\title{
Sacroiliitis and osteomyelitis caused by serotype 3 Streptococcus pneumoniae in a previously healthy adult: a case report
}

This article was published in the following Dove Press journal: Infection and Drug Resistance

\section{Yoshiro Hadano' \\ Bin Chang 2}

'Department of Infectious Diseases, St. Mary's Hospital, Kurume, Fukuoka, Japan; ${ }^{2}$ Department of Bacteriology I, National Institute of Infectious Diseases, Shinjuku-ku, Tokyo, Japan
Correspondence: Yoshiro Hadano Department of Infectious Diseases, St. Mary's Hospital, 422 Tsubukuhonmachi, Kurume, Fukuoka, 830-8543, Japan

Tel +8I 942353322

Fax +8I 942343 II 5

Email y-hadano@st-mary-med.or.jp

\begin{abstract}
Streptococcus pneumoniae is a rare cause of pyogenic sacroiliitis and osteomyelitis. A case of pneumococcal sacroiliitis and osteomyelitis is reported herein. The patient was a previously healthy 54-year-old man with a 3-day history of fever and a 3-week history of increasing right-side thigh pain. Computed tomography showed bone destruction of the right sacroiliac joint with perifocal abscess formation in the right iliac muscle. The patient was diagnosed with pyogenic sacroiliitis and osteomyelitis with a right iliac muscle abscess. Mucoid serotype 3 S. pneumoniae was obtained by culture of abscess. The patient was successfully treated with an 8-week course of antibiotics.
\end{abstract}

Keywords: Streptococcus pneumoniae, pyogenic sacroiliitis, osteomyelitis, serotype 3, invasive pneumococcal diseases

\section{Introduction}

Streptococcus pneumoniae is commonly found in nasopharyngeal mucosa of healthy people and can cause various diseases, such as pneumonia and meningitis. ${ }^{3}$ The bacterium is a relatively uncommon cause of septic arthritis, including pyogenic sacroiliitis, which is a rare manifestation of invasive pneumococcal diseases (IPD). ${ }^{1}$ The prognosis of IPD is influenced by both bacterial factors, such as serotype, and host characteristics, such as age, chronic cardiovascular and pulmonary diseases, diabetes mellitus, alcoholism, smoking, and immunosuppressive states. ${ }^{2,3}$ Because serotype 3 S. pneumoniae have a thick capsule, it is thought that it is highly pathogenic. ${ }^{4}$ In this report, we describe a rare case of pyogenic sacroiliitis and osteomyelitis with a right iliac muscle abscess caused by serotype $3 S$. pneumoniae in a healthy adult.

\section{Case report}

A previously healthy 54-year-old Japanese man was admitted to our hospital with a 3-week history of worsening right-side thigh pain and a 3-day history of fever. Four weeks prior to admission, the patient had a mild cough with sputum. His symptoms improved without any treatment. Three weeks prior to admission, the patient developed mild right thigh pain without trauma that did not interfere with his daily life. One week prior to admission, he came to a local clinic because of his right thigh pain, and loxoprofen was prescribed. His symptoms transiently improved with loxoprofen, but 3 days prior to admission, the patient had chills and his thigh pain worsened. He returned to the local clinic, and amoxicillin/clavulanate was prescribed. On the day of admission, he visited another community hospital and was transferred to our 
hospital on the diagnosis of right iliac muscle abscess. He denied sore throat, cough, dyspnea, and chest pain. His past history was not remarkable. The patient had no previous medical history, no use of tobacco or illicit drug, and rarely drink alcohol. He denied recent animal exposure, recent travel, or any history of allergies. On physical examination, his blood pressure was 140/60 $\mathrm{mmHg}$, pulse rate was 66 beats per minute, temperature was $38.0^{\circ} \mathrm{C}$, and respiratory rate was 20 breaths per minute with oxygen saturation of $97 \%$ on room air. The results of the physical examination were unremarkable, except for right buttock tenderness and positive right psoas sign. Laboratory data obtained on admission revealed a white blood cell count of $19,070 \mu \mathrm{L}$ with $94 \%$ neutrophils. Examination of the serum chemistry revealed the following results: blood urea nitrogen $23.9 \mathrm{mg} /$ $\mathrm{dL}$, creatinine $1.6 \mathrm{mg} / \mathrm{dL}$, albumin $2.2 \mathrm{~g} / \mathrm{dL}$, total protein $6.6 \mathrm{~g} / \mathrm{dL}$, and C-reactive protein $26.0 \mathrm{mg} / \mathrm{dL}$. Based on these findings, the patient was suspected of having right psoas abscess. CT of the pelvic area showed bone destruction of the right sacroiliac joint with perifocal abscess formation in the right iliac muscle (Figure 1A). A diagnostic CT-guided percutaneous drainage of the abscess was performed, and Gram staining of the pus revealed Gram-positive diplococcus bacteria and polymorphonuclear leukocytes (Figure 2). After an initial work up in the emergency room, his primary diagnosis was bacterial sacroiliitis and osteomyelitis with right iliac muscle abscess. Transthoracic echocardiography was performed, and the results showed no evidence of an infection. The patient was started on intravenous cefmetazole at a dose of 2 g every 12 hours. No bacterium was detected from his blood culture, but the pus culture grew S. pneumoniae. Additional examination revealed the following results: $90 \mathrm{~mm} / \mathrm{h}$ erythrocyte sedimentation rate, $56 \mathrm{mg} / \mathrm{dL}$ IgM (within normal range 33-183 mg/dL), $1450 \mathrm{mg} / \mathrm{dL}$ IgG (within normal range 861-1,747 mg/dL), $148 \mathrm{mg} / \mathrm{dL} \mathrm{C} 3$ (within normal range 73-138 mg/dL), $40 \mathrm{mg} / \mathrm{dL} \mathrm{C} 4$ (within normal range $11-31 \mathrm{mg} / \mathrm{dL}$ ), and $70 \mathrm{mg} / \mathrm{dL}$ CH50 (within normal range $25-48 \mathrm{mg} / \mathrm{dL}$ ). Human immunodeficiency virus antibody, $\mathrm{M}$ protein, and Bence Jones protein were negative. CT of the abdomen showed no evidence of any abdominal finding including spleen. Serum protein fraction came back with an acute inflammation pattern without $\mathrm{M}$ protein. Mucoid S. pneumoniae was obtained by culturing of abscess sample. Serotyping of $S$. pneumoniae was performed by the capsular swelling method using pneumococcal antisera (Statens Serum Institute, Copenhagen, Denmark) and the stain was determined to be serotype 3. Susceptibility of $S$. pneumoniae to antibiotics was analyzed using
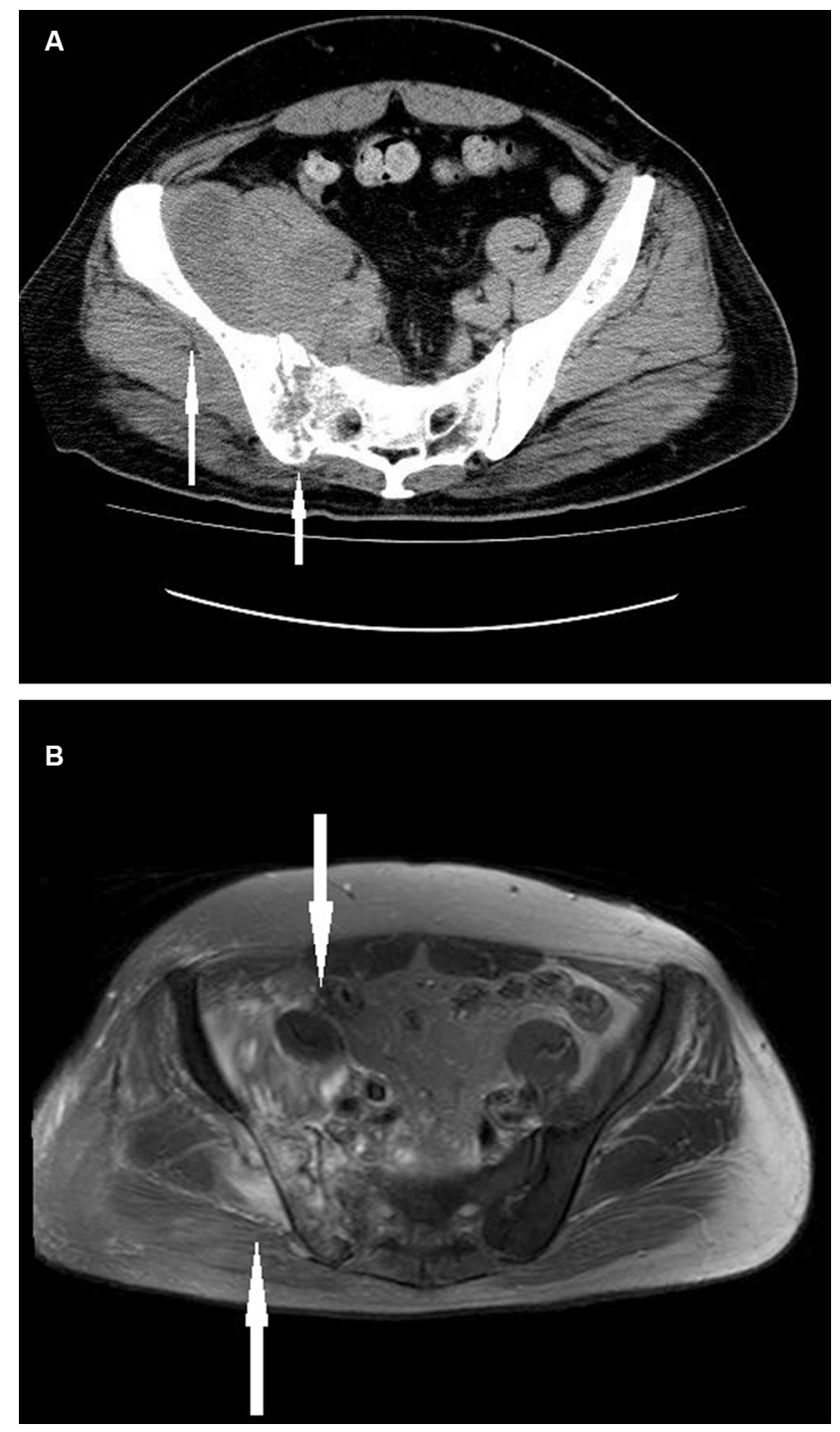

Figure I CT and MRI of the pelvic area.

Notes: (A) Bone destruction of the right sacroiliac joint with perifocal abscess formation in the right iliac muscle (white arrows); (B) MRI of the pelvic area, axial short-TI inversion recovery, which shows diffuse thickening and increased signal intensity in the right iliacus muscle, as well as edema in the fat surrounding the muscle (white arrows).

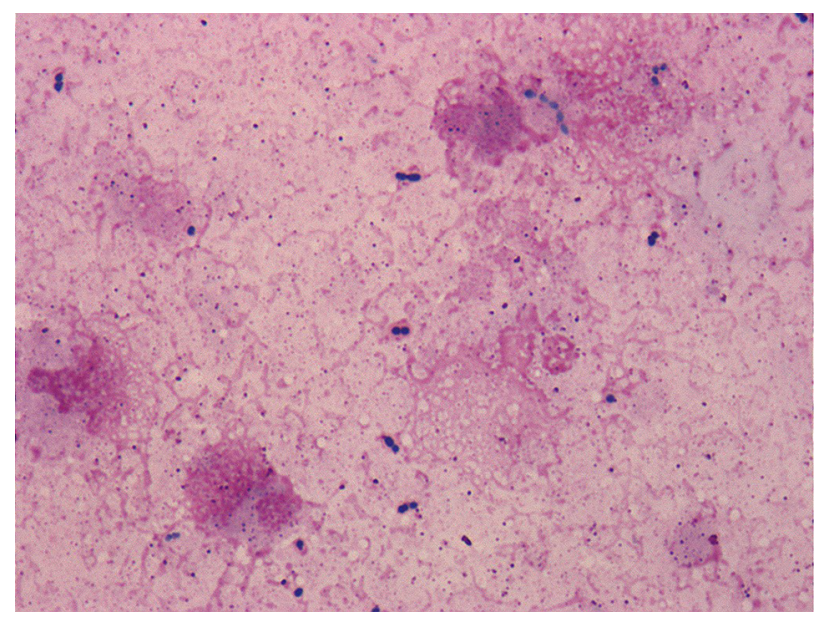

Figure 2 Gram staining of the pus $(\times 1000)$. 
Table I Susceptibility testing of the isolated Streptococcus pneumoniae

\begin{tabular}{ll}
\hline Antimicrobial agent & MIC $(\mathbf{\mu g} / \mathbf{m L})$ \\
\hline Penicillin & 0.03 \\
Ampicillin & 0.06 \\
Ceftriaxone & 0.25 \\
Meropenem & 0.015 \\
Erythromycin & 4 \\
Tosufloxacin & $\leq 0.12$ \\
Clindamycin & $\geq 8$ \\
Vancomycin & 0.5 \\
Sulfamethoxazole/trimethoprim & $\leq 0.25$ \\
\hline
\end{tabular}

Abbreviation: MIC, minimal inhibitory concentration.

the broth microdilution method according to a protocol of the Clinical and Laboratory Standards Institute. Minimal inhibitory concentration breakpoints were defined according to the Clinical and Laboratory Standards Institute (M100) criteria. The $S$. pneumoniae strain exhibited susceptibility to penicillin, ampicillin, ceftriaxone, meropenem, tosufloxacin, vancomycin, and trimethoprim/sulfamethoxazole, but was resistant to clindamycin (Table 1). On day 4, magnetic resonance imaging of the pelvic area revealed increased signal intensity of the right sacroiliac joint and surrounding bone, and diffuse thickening and effusion surrounding the muscle (Figure 1B). On day 5, we attempted to perform de-escalation. The patient was switched to treatment with ampicillin at a dose of $2 \mathrm{~g}$ every 6 hours on the basis of the susceptibility test because his renal function had improved. $\mathrm{He}$ was given levofloxacin $500 \mathrm{mg}$ orally because of the drug eruption due to ampicillin on day 25 . The patient was successfully treated with an 8-week course of antibiotics. No evidence of a relapse of the infection was noted at the 6-month follow-up.

\section{Ethics statement}

Written informed consent has been obtained from the patient to obtain their results and to publish the case details with accompanying images.

\section{Discussion}

Pyogenic sacroiliitis is a rare infectious diseases, representing $1 \%-2 \%$ of all cases of septic arthritis, most frequently in children and young adults. ${ }^{5}$ The risk factors for pyogenic sacroiliitis include being intravenous drug user, having infections in other organs systems, such as urinary tract infection, and pregnancy. ${ }^{6}$ According to previous reports, adult patients are more likely to have atypical presentation,

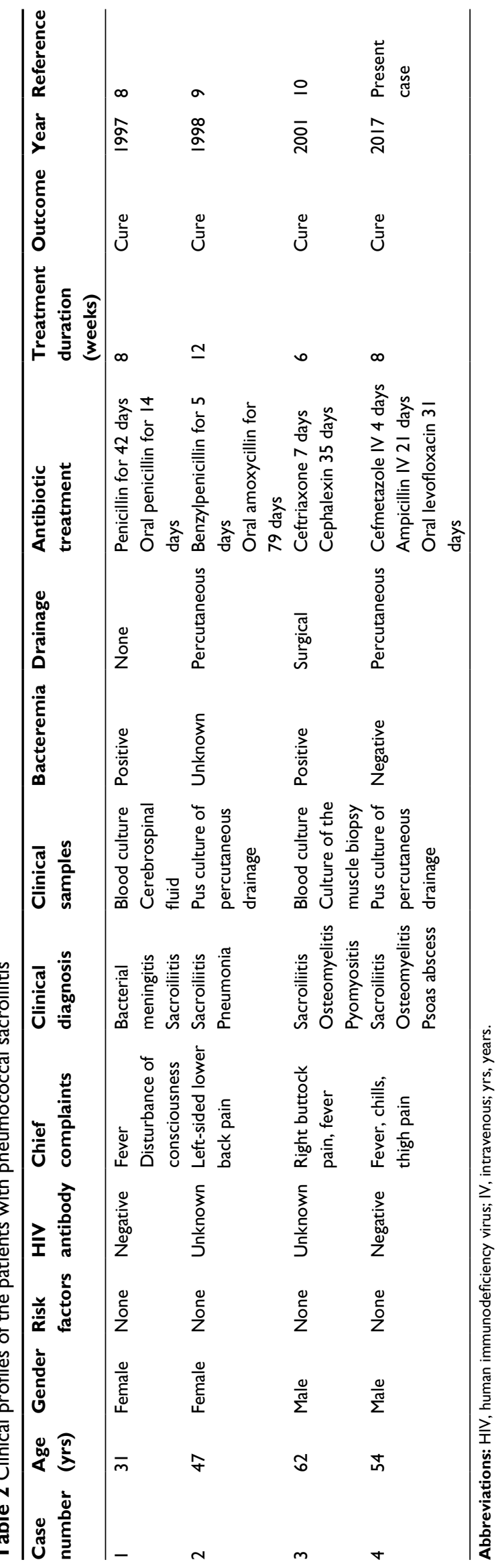


concurrent infections, and local complication. ${ }^{6,7}$ Delay in the diagnosis can lead to additional complication such as osteomyelitis, abscess formation, and bacteremia. Based on previous studies, bacteremia is documented in $71 \%$ of adults with pneumococcal septic arthritis. ${ }^{1}$ In this case, we believe that the cause of the sacroiliitis, osteomyelitis, and iliopsoas abscess was the secondary infection as a result of primary bacteremia. Although the results of the blood cultures were negative, this patient had chills, and amoxicillin/clavulanate were prescribed before admission to our hospital. Treatment options include antibiotic therapy alone, CT-guided drainage, or surgical drainage. The duration of antimicrobial treatment is at least 4-8 weeks. $^{6} S$. pneumoniae infrequently causes bacterial sacroiliitis. A review of pneumococcal septic arthritis showed $2.8 \%$ of adult patients with pneumococcal septic arthritis have sacroiliac involvement. ${ }^{7}$ In addition, three cases of pneumococcal sacroiliitis in adult have been reported in the literature (Table 2). ${ }^{8-10}$ These cases include a 31 -year-old woman who presented with a fever, depressed level of consciousness, and right buttock and thigh pain, which was later proven to be caused due to $S$. pneumoniae by blood cultures; ${ }^{8}$ a 47-year-old woman who presented with a 4-week history of left-sided lower back pain which was proven to be caused due to $S$. pneumoniae by CT-guided drainage; 9 and a 62 -yearold who presented with a 3-day history of right buttock pain which was proven to be caused due to $S$. pneumoniae by blood cultures, muscle biopsy, and surgical drainage. ${ }^{10}$ In the present case, the patient did not have an immunodeficiency or chronic illness. Interestingly, all of the patients had no risk factor for IPD. S. pneumoniae is covered by a capsule, which is a very important factor for its pathogenicity. In the case presented herein, serotype $3 \mathrm{~S}$. pneumoniae was isolated from the lesion and determined as the causative pathogen. Serotype $3 S$. pneumoniae strains always have a characteristically thicker capsule, show mucoid formation on blood agar plates, possess greater virulence, and have a higher mortality rate when compared to other serotype strains. ${ }^{3,4,11}$ In the present case, the treatment duration was 8 weeks with no evidence of a relapse of the infection during the 6-month follow-up. We believe an adequate treatment duration leads to successful treatment, even in the case of serotype 3 infection.

\section{Conclusion}

In conclusion, we reported a case of serotype 3 pneumococcal sacroiliitis and osteomyelitis with right iliac muscle abscess in a healthy adult. It is important to keep in mind that bacterial sacroiliitis and osteomyelitis due to $S$. pneumoniae are rare, but can occur even in a previously healthy adult. Delay in the diagnosis can lead to additional complication such as osteomyelitis, abscess formation, and bacteremia.

\section{Disclosure}

The authors report no conflicts of interest in this work.

\section{References}

1. Ross JJ, Saltzman CL, Carling P, Shapiro DS. Pneumococcal septic arthritis: review of 190 cases. Clin Infect Dis. 2003;36(3):319-327.

2. Alanee SR, McGee L, Jackson D, et al. Association of serotypes of Streptococcus pneumoniae with disease severity and outcome in adults: an international study. Clin Infect Dis. 2007;45(1):46-51.

3. Henriques-Normark B, Tuomanen EI. The pneumococcus: epidemiology, microbiology, and pathogenesis. Cold Spring Harb Perspect Med. 2013;3(7):pii: a010215.

4. Jansen AG, Rodenburg GD, van der Ende A, et al. Invasive pneumococcal disease among adults: associations among serotypes, disease characteristics, and outcome. Clin Infect Dis. 2009;49(2):e23-e29.

5. Hodgson BF. Pyogenic sacroiliac joint infection. Clin Orthop Relat Res. 1989;(246):146-149.

6. Slobodin G, Rimar D, Boulman N, et al. Acute sacroiliitis. Clin Rheumatol. 2016;35(4):851-856.

7. Wu MS, Chang SS, Lee SH, Lee CC. Pyogenic sacroiliitis - a comparison between paediatric and adult patients. Rheumatology (Oxford). 2007;46(11):1684-1687.

8. Pinson AG, Jolles PR, Balkissoon AR. Pneumococcal sacroiliitis. South Med J. 1997;90(6):649-652.

9. O'Brien CM, Darley ES, Kelly AJ, Nelson IW. Septic sacroiliitis: an unusual causative organism in a rare condition. Int $J$ Clin Pract. 1998;52(3):206-207.

10. Baddour VT, Hugenberg ST, Brandt KD, Yang EL. Pneumococcal pyomyositis: a patient with diagnostic problems and complications. J Clin Rheumatol. 2001;7(1):21-29.

11. Weinberger DM, Harboe ZB, Sanders EA, et al. Association of serotype with risk of death due to pneumococcal pneumonia: a meta-analysis. Clin Infect Dis. 2010;51(6):692-699.
Infection and Drug Resistance

Publish your work in this journal

Infection and Drug Resistance is an international, peer-reviewed openaccess journal that focuses on the optimal treatment of infection (bacterial, fungal and viral) and the development and institution of preventive strategies to minimize the development and spread of resistance. The journal is specifically concerned with the epidemiology of antibiotic

\section{Dovepress}

resistance and the mechanisms of resistance development and diffusion in both hospitals and the community. The manuscript management system is completely online and includes a very quick and fair peerreview system, which is all easy to use. Visit http://www.dovepress.com/ testimonials.php to read real quotes from published authors. 